\title{
Meningiomas del surco olfatorio. Consideraciones del abordaje endonasal endoscópico extendido transcribiforme
}

Omar López Arbolay, MD, PhD¹, Marlon Ortiz Machín MD1, Mayela Marrero Aliño PscD¹, Livan Santana Chil MD1

1 Hospital Clínico Quirúrgico “Hermanos Ameijeiras”. San Lázaro \# 701 Centro Habana. La Habana. Cuba.

Rev. Chil. Neurocirugía 42: 31-36, 2016

\begin{abstract}
Resumen
Los meningiomas del surco olfatorio representan el 10\% de los meningiomas intracraneales, se originan de la lámina cribosa del etmoides, la sutura fronto-esfenoidal y el plano esfenoidal. Son tumores en su mayoría benignos y potencialmente curables, la recurrencia ocurre en grado variable siendo el grado de resección quirúrgica el predictor más importante de recurrencia. En este artículo se exponen los resultados alcanzados con el abordaje endonasal endoscópico extendido transcribiforme en pacientes con meningiomas del surco olfatorio en el servicio de neurocirugía del hospital clínico quirúrgico Hermanos Ameijeiras. La serie fue de 12 pacientes donde la cefalea, la anosmia y los trastornos neuropsicológicos fueron los síntomas predominantes. Los tumores tuvieron un tamaño $\geq$ a $6 \mathrm{~cm}$ en el $50 \%$ de los casos y con el abordaje endonasal endoscópico extendido transcribiforme se alcanzó una resección total con Simpson I en el 92\% de los enfermos. Los límites del abordaje endonasal endoscópico en la fosa anterior se encuentran en constante extensión, siendo el abordaje endonasal endoscópico extendido transcribiforme la opción ideal y prometedora para los pacientes con Meningiomas del surco olfatorio.
\end{abstract}

Palabras clave: Meningeoma, surco olfatorio,abordaje endoscópico.

\begin{abstract}
Olfactory groove meningiomas represent $10 \%$ of intracranial meningiomas, originate from cribriform plate of ethmoid, frontal and sphenoid suture and the sphenoid plane. They are mostly benign and potentially curable tumors, the recurrence occurs in varying degree and the extent of surgical resection is the most important predictor of this recurrence. This article presents the results achieved with the transcribiform extended endoscopic endonasal approach in patients with meningiomas of olfactory groove in neurosurgery department of the "Hermanos Ameijeiras" hospital. The series was of 12 patients where headache, anosmia, and neuropsychological disorders were the predominant symptoms. The tumors had a size $\geq 6 \mathrm{~cm}$ on $50 \%$ of the cases and with transcribiform extended endoscopic endonasal approach was reached total removal in $92 \%$ (Simpson I) of the patients. The limits of endoscopic endonasal approach for anterior fossa are in constant expansion, being the transcribiform extended endoscopic endonasal approach the ideal and promising option for patients with olfactory groove meningiomas.
\end{abstract}

Key words: Meningioma, olfactory groove,endoscopic approach.

\section{Introducción}

Los meningiomas del surco olfatorio representan el $10 \%$ de los meningiomas intracraneales, se originan en el área de la lámina cribosa del etmoides, la sutura fronto-esfenoidal y el plano es- fenoidal ${ }^{1}$. A pesar de ser tumores en su mayoría benignos y potencialmente curables, la recurrencia en 10 años en las diferentes series varía entre un 10 y un $41 \%$, siendo el grado de resección quirúrgica el predictor más importante de recurrencia ${ }^{2,3}$. Este índice de recu- rrencia ha sido atribuido a la dificultad de remover las células tumorales que invaden la base craneal y senos paranasales con los abordajes tradicionales ${ }^{4}$. Debido a su localización subfrontal, alejado de áreas neurológicas de significativa elocuencia, estos tumores 
tienen un comportamiento clínico larvado y silencioso, expresándose cuando el tumor alcanza un tamaño considera$b e^{5}$. Es frecuente apreciar hiperostosis en el hueso adyacente, así como la invasión del tumor al seno etmoidal y a la cavidad nasal en un $15 \%$ de los ca$\operatorname{sos}^{6-11}$. Son más frecuentes en el sexo femenino entre la quinta y sexta décadas de la vida; y en series como la de Hentschel-DeMonte y Turazzi en más del $50 \%$ de los casos los tumores tuvieron diámetros superiores a $6 \mathrm{~cm}$ en el momento del diagnóstico ${ }^{12,13}$. Cuando los diámetros tumorales son pequeños, los nervios olfatorios pueden ser identificados en los márgenes laterales del tumor y pueden ser preservados, sin embargo, en grandes tumores, los nervios olfatorios están afinados, a tal grado que su preservación es casi imposible $^{14}$. La vascularización primaria es a través de las arterias etmoidales anteriores y posteriores, las cuales son ramas de la arteria oftálmica, aunque también tienen suplencia de ramos meníngeos de la arteria oftálmica directamente, arterias cerebrales anteriores, arteria comunicante anterior, así como ramos de la arteria meníngea media. Existen frecuentemente anastomosis entre las arterias etmoidales y los ramos meníngeos de la arteria carótida interna y de la arteria oftálmica propiamente dicha, esto hace que la embolización del tumor siempre sea un riesgo en la preservación de la arteria oftálmica en su punto crítico donde origina a la arteria central de la retina y por tanto, la conservación de la visión ${ }^{14-15}$. Las arterias fronto-polares y otras pequeñas ramas de la arteria cerebral anterior pueden ser sacrificadas sin consecuencias, si están adheridas a la cápsula tumoral en el polo posterior del mismo ${ }^{15}$. El síntoma más frecuente es la cefalea aislada o en combinación con trastornos de conducta, anosmia y disturbios visuales. La hipertensión intracraneal y las convulsiones son menos frecuentes inicialmente. Puede presentarse como un trastorno psiquiátrico de larga fecha de evolución antes de aparecer el resto de los síntomas neurológicos ${ }^{16,17,18,19}$. El síndrome de Fóster-Kennedy, clásicamente descrito como papila pálida ipsilateral con papiledema contralateral es muy raro, Simon reportó solo un caso en su serie de 40 pacientes $^{20}$.

Los abordajes quirúrgicos más frecuentes para tratar estas lesiones son el abordaje bifrontal transbasal uni o bilateral y el abordaje pterional. Estos han sido favorecidos con las técnicas de base de cráneo, a través de orbitotomías mediales o laterales y la resección de parte del cigoma ${ }^{22,23}$.

Con el surgimiento y desarrollo de la cirugía endoscópica a la hipófisis y región selar; y su extensión a otras áreas de la base craneal, surgieron abordajes como: el transcribiforme. Abordaje que recientemente ha sido de gran interés para la resección de meningiomas de la fosa craneal anterior; permitiendo un excelente acceso a la base tumoral y la posibilidad de realizar un Simpson 1 , tópico que aun continúa en discusión $^{28-31}$

El objetivo de este artículo es exponer los resultados alcanzados con el abordaje endonasal endoscópico extendido transcribiforme en pacientes con meningiomas del surco olfatorio en el servicio de neurocirugía del hospital clínico quirúrgico Hermanos Ameijeiras desde el año 2010 hasta la actualidad.

\section{Pacientes y Método}

Se realizó un estudio descriptivo de los pacientes con diagnóstico de Meningioma del Surco Olfatorio, operados a través de un abordaje endonasal endoscópico extendido transcribiforme, en el Hospital Clínico Quirúrgico Hermanos Ameijeiras en el período del 2010 hasta la actualidad.

\section{Criterios de inclusión}

Pacientes con clínica y confirmación imagenológica de Meningioma del Surco Olfatorio.

El estudio imagenológico incluyó tomografía axial contrastada de cráneo, así como imagen de resonancia magnética (IRM) de cráneo con Gadolinium. Previo a la cirugía todos los pacientes fueron evaluados por un equipo interdisciplinario conformado por neuropsicólogos, neurooftalmólogos y neurocirujanos.

El estudio se centró en el análisis de las siguientes variables: características clínicas, imagenológicas (según IRM), grado de resección tumoral según escala de Simpson y complicaciones quirúrgicas.

La evaluación Neuropsicológica se basó en una batería de pruebas conformada por: Test Cognitivo de Montreal (Evaluación cognitiva global) ${ }^{35}$, TBA (Test breve de atención), Test de aprendizaje de memoria verbal de Hopkins, Test de Wisconsin, Figura compleja de Rey (copia y memoria visuoespacial), Test de reconocimiento de rostros de Benton y Test de fluidez verbal, semántica y fonológica ${ }^{36,37}$.

La técnica quirúrgica empleada fue el Abordaje Endonasal Endoscópico Extendido Transcribiforme ${ }^{33,34}$, utilizando como principios básicos el abordaje binarial, para permitir a dos cirujanos realizar una amplia resección ósea de la base craneal que incluye la lámina cribosa y el plano esfenoidal, pudiéndose extender a la cara anterior y piso de la silla turca si fuese necesario en el plano sagital. En el plano coronal se identificaron ambas láminas papiráceas, permitiendo un abordaje extendido entre ambas paredes mediales orbitales.

Se realizó una técnica a cuatro manos creando un amplio corredor quirúrgico. Se emplearon básicamente cuatro endoscópios rígidos, 0, 30, 45 y 70 grados (Hopkins II), de $18 \mathrm{~cm}$ de longitud, $4 \mathrm{~mm}$ de diámetro y un módulo vídeo -endoscópico de alta definición (Karl Storz). El grado de resección tumoral fue evaluado por TAC a las 24 horas de operado el paciente y por IRM a los tres meses, se utilizó la escala de Simpson'1.

Todos los pacientes fueron informados sobre su enfermedad y sobre el proceder quirúrgico que se les realizaría y se les solicitó su consentimiento antes de ser incluidos en la serie, respetándose los principios fundamentales de la ética médica.

\section{Resultados (Tabla 1)}

\section{Discusión}

Tradicionalmente los Meningiomas del Surco Olfatorio han sido abordados por vía transcraneal a través de craneotomías frontales uni o bilaterales (bifrontales), así como por vías más oblicuas a través de craneotomías pterionales o fronto-temporales, estas técnicas han sido favorecidas con los abordajes de base de cráneo, añadiéndose orbitotomías mediales en el caso de los abordajes anteriores así como osteotomías orbito-cigomáticas en el caso de las vías laterales, permitiendo descender más el abordaje para atacar la base de implantación tumoral, como parte de 
Tabla 1.

Caracterización de la serie y resultados del tratamiento endonasal endoscópico extendido transcribiforme a los meningiomas del surco olfatorio

\begin{tabular}{|c|c|}
\hline Cantidad de pacientes & $\mathrm{n}=12$ \\
\hline Edad Promedio & 52,6 años \\
\hline Relación sexo F/M & $1 / 1$ \\
\hline Forma clínica de presentación & $\begin{array}{l}\text { Cefalea }(91,6 \%) \text {, Anosmia }(50 \%) \text {, Trastornos Neuropsicológicos }(100 \%) \text {, Convul- } \\
\text { siones }(58 \%)\end{array}$ \\
\hline $\begin{array}{l}\text { Evaluación Neuropsicológica } \\
\text { Pre y Postquirúrgico (3 meses) }\end{array}$ & $\begin{array}{l}\text { Trastornos de Memoria: Pre }(91,6 \%), \text { Post }(50 \%) \\
\text { Trastornos de Atención: Pre }(100 \%) \text {, Post }(41.6 \%) \\
\text { Trastornos de Orientación: Pre }(50 \%), \text { Post }(25 \%) \\
\text { Trastornos Neurocomportamentales: Pre }(91,6 \%) \text {, Post }(83,3 \%)\end{array}$ \\
\hline Tamaño tumoral & $2-4 \mathrm{~cm}(16 \%), 4-6 \mathrm{~cm}(32 \%), \geq 6 \mathrm{~cm}(50 \%)$ \\
\hline Resección Tumoral & Simpson I: 11 (pacientes 92\%) \\
\hline Complicaciones & Fístula de LCR (8\%), TVP (8\%), ITU (8\%) \\
\hline Escala de Karnofsky al egreso & $90-100$ ptos (8 pacientes), 80 ptos ( 4 pacientes) \\
\hline Escala de Glasgow a los 6 meses & Buena recuperación (10 pacientes), Incapacidad moderada (2 pacientes) \\
\hline
\end{tabular}

los principios de la cirugía de los meningiomas ${ }^{8,34}$. En las diferentes series reportadas desde el año 1996 hasta el 2003, utilizando abordajes tradicionales para el tratamiento de estos tumores se plantea un índice de resección total de un $98 \%$, sin embargo, se refieren a la resección macroscópica total sin tener en cuenta la escala de Simpson a pesar de haber sido descrita la misma en el año $1957^{5}$. En un estudio retrospectivo de 59 pacientes sobre meningiomas del surco olfatorio operados por técnicas transcraneales tradicionales, publicado en la revista neurosurgical focus del año 2011, se realiza un análisis del grado de resección tumoral aplicando la escala de Simpson, obteniéndose los siguientes resultados: Simpson 1 (23,7\%), Simpson 2 (64,4\%), Simpson $3(6,8 \%)$, Simpson $4(5,1 \%)$ y Simpson $5(0 \%)$. Estos resultados pueden explicar el elevado índice de recidiva tumoral de hasta un $40 \%$ entre 5 y 10 años posterior al tratamiento quirúrgico secundario a la persistencia de restos tumorales hacia la lámina cribosa del etmoides $^{8,21}$. Con el desarrollo de la cirugía Endonasal Endoscópica a la hipófisis y la posterior extensión de los abordajes a otras áreas de la base craneal, surge el abordaje endonasal endoscópico extendido transcribiforme, el cual permite un excelente acceso a la base craneal anterior desde una perspectiva inferior y cumple con los princi- pios de la cirugía de los meningiomas: abordarlos por la base, desvascularización temprana, vaciamiento y disección subaracnoidea de la cápsula 2,7,14,24,28,32. La identificación temprana de las arterias etmoidales así como la resección precoz de toda la base de implantación es una ventaja exclusiva del abordaje endoscópico transcribiforme (Figuras 2, 3, 4). Entre los años 2010-2015 se operaron por vía endoscópica un total de 12 pacientes con meningiomas del surco olfatorio (Tabla 1) no observándose un predominio del sexo femenino como refieren otros autores (69\%) $5,21,24,28,34$. La edad promedio fue de 52,6 años, similar a las demás series ${ }^{5,32}$. La forma clínica de presentación más frecuente fue la cefalea $(91,6 \%)$, lo que puede guardar relación con el gran tamaño tumoral en el momento del diagnóstico, aunque cabe señalar que en un caso donde el tumor ocupaba prácticamente toda la fosa craneal anterior con diámetros superiores a los $6 \mathrm{~cm}$, nunca presentó cefalea. La evaluación Neuropsicológica detallada arrojó que todos los pacientes presentaron algún daño cognoscitivo en el preoperatorio: trastornos de atención (100\%), memoria $(91,6 \%)$ y orientación $(50 \%)$, apreciándose una mejoría en la evaluación postoperatoria; atención (41,6\%), memoria $(50 \%)$ y orientación $(25 \%)$. Los Trastornos neuro-comportamentales fueron muy frecuentes en la evaluación preoperatoria $(91,6 \%)$, apreciándose una ligera mejoría de los mismos después de la cirugía (83,3\%). Tabla 1. En la literatura se reconoce que los pacientes con meningiomas del surco olfatorio presentan trastornos conductuales en más de un $55 \% \%^{5,21,32}$, sin embargo, no se encontró en la revisión realizada una evaluación neuropsicológica detallada a pacientes con meningiomas del surco olfatorio. Somos del criterio que estos trastornos pueden ser identificados de forma precoz en pacientes aparentemente asintomáticos, por tanto, la evaluación neuropsicológica a nuestro criterio es fundamental incluso para objetivar el daño que está originando el tumor por la compresión y atrofia encefálica. La anosmia estuvo presente en el $50 \%$ y está relacionado con el daño que origina el tumor sobre los nervios olfatorios, aunque no es frecuente en tumores menores de 3 $\mathrm{cm}$ de diámetro ${ }^{5}$. Las convulsiones se presentaron en un $58 \%$, superior a lo reportado en otras series donde representan un $35 \%{ }^{21}$, esto puede guardar relación con el gran tamaño tumoral en el momento del diagnóstico. Llama la atención que en $50 \%$ de los casos el diámetro tumoral fue superior a $6 \mathrm{~cm}$, siendo clasificados como meningiomas gigantes del surco olfatorio. En otras series se observa un predominio de tumores con diámetros entre 4-6 cm, lo cual manifiesta un posible diagnóstico 
tardío ${ }^{5,21,34}$. En cuanto a la resección tumoral se logró un Simpson I en un 92\% de los casos, solo en un caso se dejó un fragmento tumoral que encarcelaba ambas arterias cerebrales anteriores, sin embargo, se retiró toda la base de implantación, el hueso, la duramadre y las principales arterias nutricias; por tanto, pensamos que en este caso es difícil de evaluar según la escala de Simpson el grado de resección. Estos resultados contrastan de forma significativa con el grado de resección según la escala de Simpson a través de abordajes transcraneales donde se logró un Simpson I en sólo el $23,7 \%$ de la serie ${ }^{21}$, predominando el Simpson II $(64,4 \%)$. En otras series quirúrgicas con abordajes endoscópicos como la reportada en la Universidad de Pittsburg entre los años 2002-2005, se reporta un Simpson I en el $83 \%$ de los pacientes ${ }^{34}$ y en otras un $66,7 \% \%^{32}$. Se han descrito factores que imposibilitan la resección total (Simpson I) como: la presencia de grandes calcificaciones, la ausencia del manto cortical, el encarcelamiento vascular, el diámetro mayor de $40 \mathrm{~mm}$ y el crecimiento lateral más allá de la $1 / 2$ del techo orbitario $0^{5,21,28,32,33,34}$. En cuanto a las complicaciones quirúrgicas, la fístula de LCR estuvo presente en el $8 \%$ de los pacientes (1 caso), la cual fue resuelta con procedimientos endoscópicos endonasales, sin necesidad de craneotomía.

La fístula de LCR como resultado del gran defecto de la base craneal y la dificultad en el proceso de reparación quirúrgica ha sido el argumento que de forma tradicional han planteado muchos autores para abordar estos tumores por vías transcraneales, prefiriendo un Simpson $I I^{1,2,5,14,20}$. Con el surgimiento de la filosofía de los colgajos vascularizados en el proceso de reparación

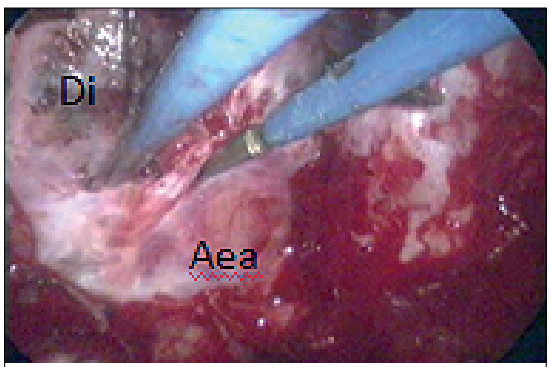

Figura 2. Abordaje Endoscópico Transcribiforme, exéresis de duramadre infiltrada y devascularización. Di. Duramadre infiltrada; Aea. Arteria etmoidal anterior. de la base craneal, los resultados se han transformado de forma dramática, con incidencias de fístulas de un $40 \%$ inicialmente a un $5,4 \%$ después del surgimiento del colgajo nasoseptal ${ }^{32,34}$. Somos del criterio que la reparación de la base craneal multicapa: tejido graso, fascia lata, hueso septal, colgajo nasoseptal y capa de Gelfoam, fijados con balón de sonda foley, fueron determinantes en los resultados. Las demás complicaciones fueron una trombosis venosa profunda y una infección del tracto urinario, las cuales fueron tratadas respectivamente. (Tabla 1). Las complicaciones como edema frontal, infarto venoso, hematomas, infección de la herida quirúrgica, neumoencéfalo, con una mortalidad cercana al $5 \%$, son más frecuentes en abordajes transcraneales secundario a la retracción cerebral, a la manipulación de estructuras neurovasculares, senos venosos y neumáticos, que frecuentemente están presentes en estos tipos de accesos ${ }^{22}$. El grado de reincorporación fue evaluada según las escala de Karnofky al egreso y la Escala de Glasgow para resultados a los 6 meses (Tabla 1).Todos los pacientes alcanzaron un Karnofsky entre 80-100 puntos al egreso y sólo 2 pacientes presentaron una incapacidad moderada a los 6 meses de operados, estos resultados son similares a los reportados por la literatura ${ }^{21}$

\section{Nuestra filosofía}

La palabra límite del romano limes se refirió inicialmente para separar grandes territorios, sin embargo, es un término que frecuentemente se utiliza en las matemáticas, física, química, geografía y anatomía como medida de tendencia, aunque en determinadas ciencias como la psicología, sociología y pedagogía, la palabra límite puede significar restricción del pensamiento. En cuanto a los límites establecidos para el abordaje endonasal endoscópico para los meningiomas del surco olfatorio se encuentran: el diámetro menor de $40 \mathrm{~mm}$, no grandes calcificaciones, no encarcelamiento vascular, crecimiento medial a la $1 / 2$ del techo orbitario, poco edema vasogénico asociado, presencia de un manto cortical entre

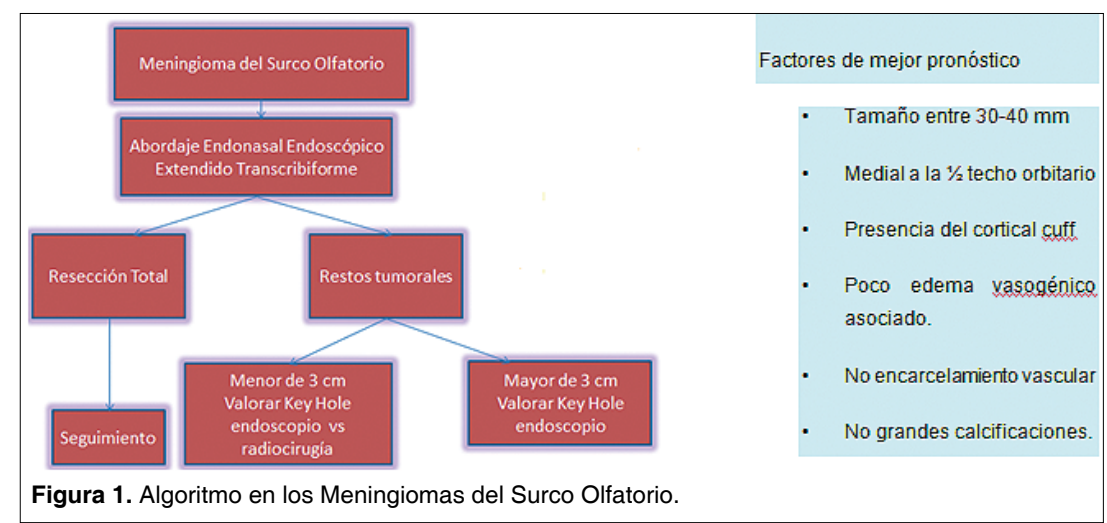

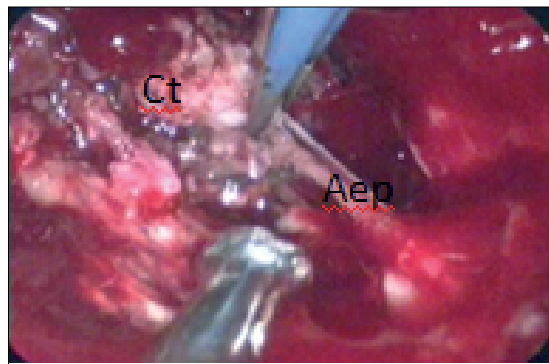

Figura 3. Abordaje Endoscópico Transcribiforme, vaciamiento tumoral y devascularización. Ct. Centro tumoral; Aep. Arteria etmoidal posterior.

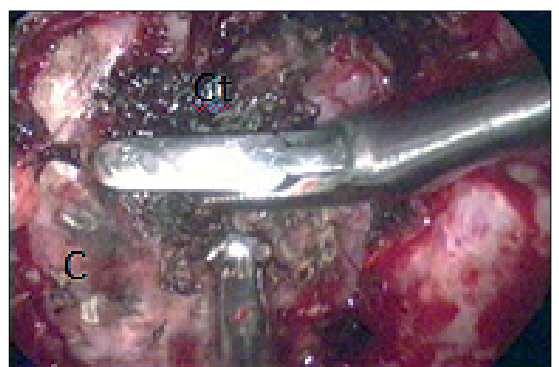

Figura 4. Abordaje Endoscópico Transcribiforme, vaciamiento y reducción tumoral. C. Cápsula tumoral; Ct. Centro tumoral. 
el tumor y las estructuras vasculares así como crecimiento hacia la cavidad nasal ${ }^{21}, 32,33,34$. Somos del criterio que todos estos factores no limitan el abordaje endonasal endoscópico, más bien pueden ser definidos como factores pronósticos. El $50 \%$ de los pacientes operados en nuestra serie presentaban diámetros tumorales superiores a $6 \mathrm{~cm}$, lográndose en la mayoría de los casos una resección total, Simpson I. (Tabla 1). El abordaje endoscópico desde una perspectiva inferior, permite atacar el tumor de forma directa por la base, con una devascularización temprana, per- mitiendo realizar la exéresis de la duramadre y el hueso infiltrado, siguiendo los principios de la cirugía de los meningiomas. El vaciamiento tumoral reduce considerablemente el tamaño tumoral central y de esta forma permite atraer hacia la línea media la porción lateral del tumor localizada más allá de la mitad del techo orbitario. Posteriormente se realiza una disección subaracnoidea cuidadosa, utilizando pulsos de suero, instrumentos romos y filosos. Los restos tumorales mayores de $3 \mathrm{~cm}$ pueden ser valorados para realizar un abordaje key hole endoscópico transciliar y a los menores de $3 \mathrm{~cm}$ se le puede ofrecer la opción de radiocirugía como alternativa de tratamiento. (Figura 1). Los límites del abordaje endonasal endoscópico en la fosa anterior se encuentran en constante extensión, así lo demuestra un reciente trabajo sobre la Orbitotomía Súpero-medial ${ }^{38}$ como extensión del abordaje endonasal endoscópico en fosa anterior más allá de las láminas papiráceas $^{38}$

\section{Recibido: 02 de enero de 2016 Aceptado: 03 de marzo de 2016}

\section{Bibliografía}

1. Simpson D (1957). The recurrence of intracranial meningiomas after surgical treatment. J NeurolNeurosurg Psychiatry $20: 22.39$.

2. Obeid F, Al Mefty O. Recurrence of olfactory groove meningioma. Neurosurgery 2003; 53: 534-542.

3. Mirimanoff RO, Dosoretz DE, Linggood RM, et al. Meningioma: analysis of recurrence and progression following neurosurgical resection. J Neurosurg 1985; 62:18-24.

4. Joung H Lee. Meningiomas, diagnosis, treatment and outcome 2008; 34: 327-332.

5. Hentschel SJ, DeMonte F: Olfactorygroovemeningiomas. Neurosurg Focus 14(6): e4, 2003.

6. Bakay L, Cares HL. Olfactory meningiomas. Report on a series of twenty-five cases. Acta Neurochir (Wien) 26: 1-12, 1972.

7. Dehdashti AR, Ganna A, Witterick I, Gentili F. Expanded en-doscopic endonasal approach for anterior cranial base and su-prasellar lesions: indications and limitations. Neurosurgery 64: 677-689, 2009.

8. Lee KF, Suh JH, Lee YE, Berry RG. Meningioma of the para-nasal sinuses. Neuroradiology 17: 165-171, 1979.

9. Rubinstein $A B$, Arbit E. Intracranial meningiomaspresenting with epistaxis-case report and literature review. J-Otolaryn-gol 14: 248-250, 1985.

10. Solero CL, Giombini S, Morello G: Suprasellar and olfactory meningiomas. Report on a series of 153 personal cases. ActaNeurochir-(Wien) 67: 181-194, 1983.

11. Symon L. Olfactory groove and suprasellarmeningiomas, in Krayonbühl H (ed): Advances and Technical Standards in Neurosurgery. New York: Springer-Verlag, 1977, Vol 4, pp 67-91.

12. Spektor S, Valarezo J, Fliss DM, Gil Z, Cohen J, Goldman J, Umansky F. Olfactory groove meningiomas from neurosurgical and ear, nose, and throat perspectives: approaches, techniques, and outcomes. Neurosurgery 57: 268-280, 2005.

13. Turazzi S, Cristofori L, Gambin R, et al. The pterional approach for the microsurgical removal of olfactory groove meningiomas. Neurosurgery 1999; 45: 821-825.

14. Ojemann RG. Olfactory grove meningiomas. In: Al Mefty O.Meningiomas. New York: Raven Press, 1991: 383-394.

15. Symon L. Olfactory groove and surasellarmeningiomas. In: Kayenbuhl H, ed. Advances and Technical Standards in Neuro-surgery. New York: Springer-Verlag, 1977; 69-71.

16. Alexiou GA, Gogou P, Markoula S, Kyritsis AP (2010). Managementof meningiomas. Clin Neurol Neurosurg 112(3): 177-182.

17. d'Avella D, Salpietro FM, Alafaci C, Tomasello F (1999). Giant olfactory meningiomas: the pterional approach and its relevancefor minimizing surgical morbidity. Skull Base Surg 9(1): 23-33.

18. Cushing $\mathrm{H}$, Eisenhardt $L$ (1938). Meningiomas: their classification, regional behaviour, life history and surgical end results. Charles $C$ Thomas, Springfield, pp 250-282.

19. chirSuppl (Wien) 53: 14-1818. Hentschel SJ, Demonte F (2003). Olfactory groove meningiomas. Neurosurg Focus 14(6): Article 4.

20. Fukuyama J, Hayasaka S, Setogawa T, et al. Foster Kennedy syndrome and optociliary shunt vessels in a patient with an olfactory groove meningioma. Ophthalmologica 1991; 202: 125-131.

21. VladCiurea A, Mircea lencen Stefan, EugenRizea. Radu. Olfactory groove meningiomas. A retrospective study on 59 surgical cases. Neurosurg Rev. DOI 10.1007/s 10143-011-0353-2. 2011.

22. Nakamura M, Struck M, Roser F, Vorkapic $P$, Samii M. Olfactory groove meningiomas: clinical outcome and recurrencerates after tumor removal through the frontolateral and bi-frontal approach. Neurosurgery 60: 844-852, 2007.

23. Bakay L, Cares HL. Olfactory meningiomas. Report on a series of twenty-five cases. Acta Neurochir (Wien) 26: 1-12, 1972.

24. Derome PJ, Guiot G. Bone problems in meningiomas invadingthe base of the skull. Clin Neurosurg 25: 435-451, 1978.

25. Schaller C, Rohde V, Hassler W. Microsurgical removal of ol-factory groove meningiomas via the pterional approach. Skull Base Surg 4: 189-192, 1994.

26. Snyder WE, Shah MV, Weisberger EC, Campbell RL. Presentation and patterns of late recurrence of olfactory groove meningiomas. Skull Base Surg 10: 131-139, 2000.

27. Symon L. Olfactory groove and suprasellarmeningiomas, in Krayonbühl H (ed): Advances and Technical Standards in Neurosurgery. New York: Springer-Verlag, 1977, Vol 4, pp 67-91.

28. deDivitiis E, Esposito F, Cappabianca P, Cavallo LM, de Di-vitiis O, Esposito I. Endoscopic transnasal resection of anterior cranial fossameningiomas. Neurosurg Focus 25(6): E8, 2008.

29. Dehdashti AR, Ganna A, Witterick I, Gentili F. Expanded endoscopic endonasal approach for anterior cranial base and suprasellar lesions: 
indications and limitations. Neurosurgery 64: 677-689, 2009.

30. Greenfield JP, Anand VK, Kacker A, Seibert MJ, Singh A, Brown SM, et al. Endoscopic endonasal transethmoidal transcribriformtrans foveaethmoidalis approach to the anterior cranial fossa and skull base. Neurosurgery 66: 883-892, 2010.

31. Laufer I, Anand VK, Schwartz TH. Endoscopic, endonasal extended transsphenoidal, transplanumtranstuberculum approach for resection of suprasellar lesions. J Neurosurg 106: 400-406, 2007.

32. Kassam AB, Thomas A, Carrau RL, Snyderman CH, Vescan A, Prevedello D, et al. Endoscopic reconstruction of the cranial base using a pediclednasoseptal flap. Neurosurgery 63 (1 Suppl 1): ONS44-ONS53, 2008.

33. James K, Liu, MD, Lana D, Christiano MD, Smruti K, Patel BA.,1 R. Shane Tubbs, M.S., P.A.-C., Ph.D.Surgical nuances for removal of olfactory groove meningiomas using the endoscopic endonasal transcribriform approach.

34. Gardner Paul A, Kassam Amin B, Carrau Ricardo L, Prevedello Daniel M. Endoscopic Endonasal Resection of Anterior Cranial Base Meningiomas. Clinical Studies, Neurosurgery 63: 36-54, 2008.

35. Gallego Lozano M, Ferrandíz Hernández M, Garriga Turró Oriol. Validación del Montreal Cognitive Assessment (MoCA): Test de Cribado para el deterioro cognitivo leve. Datos preliminares. Alzheimer. Real Invest Demenc 2009. 43: 4-11.

36. Ardila A, Ostrosky Feggy, Guia para el Diagnóstico Neuropsicológico, Univeridad Internacional de Florida. EEUU. 2012.

37. Portellano Antonio J. Introducción a la Neuropsicología. Universidad Complutensi de Madrid. España. 2005.

38. Valdespeñas Ruiz Cárdenas E. Orbitotomía Súpero Medial: Nuevos Límites en el Abordaje Endonasal Expandido Transetmoidal. Tesis Doctoral. Universidad de Pittsburg 2015.

\section{Correspondencia a:}

Omar López Arbolay

arbolay@infomed.sld.cu 